BI-TP 2008/1

BNL-NT-08/1

\title{
Lattice cut-off effects and their reduction in studies of QCD thermodynamics at non-zero temperature and chemical potential
}

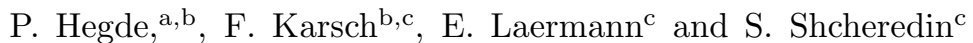 \\ ${ }^{a}$ Department of Physics and Astronomy, Stony Brook University, Stony Brook, NY 11790, USA \\ ${ }^{\mathrm{b}}$ Physics Department, Brookhaven National Laboratory, Upton, NY 11973, USA \\ ${ }^{\mathrm{c}}$ Fakultät für Physik, Universität Bielefeld, D-33615 Bielefeld, Germany
}

(Dated: October 28, 2018)

\begin{abstract}
We clarify the relation between the improvement of dispersion relations in the fermion sector of lattice regularized QCD and the improvement of bulk thermodynamic observables. We show that in the infinite temperature limit the cut-off dependence in dispersion relations can be eliminated up to $\mathcal{O}\left(a^{n}\right)$ corrections, if the quark propagator is chosen to be rotationally invariant up to this order. In bulk thermodynamic observables this eliminates cut-off effects up to the same order at vanishing as well as non-vanishing chemical potential. We furthermore show, that in the infinite temperature, ideal gas limit the dependence of finite cut-off corrections on the chemical potential is given by Bernoulli polynomials which are universal as they do not depend on a particular discretization scheme. We explicitly calculate leading and next-to-leading order cut-off corrections for some staggered and Wilson fermion type actions and compare these with exact evaluations of the free fermion partition functions. This also includes the chirally invariant overlap and domain wall fermion formulations.
\end{abstract}

PACS numbers: 11.15.Ha, 11.10.Wx, 12.38Gc, 12.38.Mh

\section{INTRODUCTION}

Numerical studies of lattice regularized QCD face the problem of lattice discretization errors which complicate the extraction of physical results in the continuum limit. This problem has been addressed ever since the formulation of a systematic improvement scheme for gauge theories by Symanzik 1]. Aside from the now widely used Symanzik improved gauge actions it also led to tree-level improved actions for the fermion sector of QCD like the Naik 2 and p4 [3] staggered fermion actions as well as the clover [4] and (truncated) perfect actions for Wilson fermions [5, [6].

The reduction of cut-off effects plays a particularly important role in studies of QCD thermodynamics where the relevant observables, like energy density or pressure, are dimension four operators and the numerical signal for these observables thus drops like the lattice spacing to the fourth power. This forces one to perform calculations on rather coarse lattices on which discretization errors can be significant. In fact, this has been observed early on in studies of the bulk thermodynamics of SU(3) gauge theories [7] and the efficiency of improved actions for studies of gauge theories at high temperature has been demonstrated [8].

In calculations on lattices with finite temporal extent $N_{\tau}$, as required in thermodynamic studies, cut-off effects obscure strongly the approach to the infinite temperature, ideal gas limit. Improvement schemes for thermodynamic calculations therefore have been developed and tested quite successfully in this limit for both the gauge as well as the fermion sector of QCD. The qualitative features of the cut-off dependence of thermodynamic observables present in this limiting case have been found to carry over to numerical calculations at finite temperatures and are significant even at temperatures as low as a few times the transition temperature to the quark-gluon plasma phase.

In this paper we will concentrate on a discussion of cut-off effects in the fermionic sector of bulk thermodynamic quantities that are given by derivatives of the QCD partition function with respect to temperature, $T$, or quark chemical potential, $\mu$. In particular the latter aspect has so far not been analyzed systematically. Some results for the $\mu$-dependence of cut-off effects have been obtained for the Naik and p4 staggered fermion actions [9, 10], truncated perfect actions [11] and for overlap fermions [12]. In fact, in the latter case the question was raised whether the introduction of a non-zero chemical potential in the overlap formalism could spoil the leading order cut-off dependence of thermodynamic quantities and could reintroduce divergences of the type discussed in early studies of QCD at finite density [13]. We proof here that this is not the case and give explicit results for the $\mu$-dependence of cut-off effects in leading and next-to-leading order in a large- $N_{\tau}$ expansion of bulk thermodynamics. We show that a non-vanishing chemical potential only modifies the expansion coefficients but does not change the structure of the expansion, i.e. actions that are improved to $\mathcal{O}\left(a^{n}\right)$ at $\mu=0$ remain improved to that order also for $\mu>0$. We will clarify the relation between the improvement of dispersion relations and the improvement of bulk thermodynamic observables and give 
explicit results for the large- $N_{\tau}$ expansion of the pressure calculated with staggered and Wilson type actions. We furthermore show that these considerations carry over to chirally invariant fermion formulations such as overlap and domain wall fermions.

We will analyze the relation between rotational invariance of quark propagators, improved dispersion relations and bulk thermodynamic observables in the next two sections. We further exemplify these results for naive staggered fermions as well as two popular improved staggered fermion actions, the Naik [2] and the p4 action [3] in section IV] In section $\mathrm{V}$ we consider fermion discretizations of the Wilson type with general couplings on a hypercube of size $(2 a)^{4}$. We finally comment on thermodynamics with overlap and domain wall fermions in section $\overline{\nabla I}$ and give our conclusions in section VII. Appendices A through D contain some useful formulas to make the paper self-contained.

\section{DISPERSION RELATIONS AND THE FREE ENERGY}

We will start our discussion of cut-off effects for free fermions which corresponds to the asymptotic infinite temperature limit of QCD by considering the simplest (naive) lattice discretization scheme used for the fermion sector of QCD. This will already demonstrate the main features of our analysis. For simplicity we also will suppress color and flavor factors, which are just multiplicative in the infinite temperature limit.

The partition function for free fermions at temperature $T$ and for non-vanishing chemical potential $\mu$, defined on a lattice of size $N_{\sigma}^{3} N_{\tau}$, is given by

$$
Z(V, T, \mu, m)=\int \prod_{x} \mathrm{~d} \bar{\psi}_{x} \mathrm{~d} \psi_{x} \mathrm{e}^{-S_{F}},
$$

where $\left(N_{\sigma} a\right)^{3}=V$ and $N_{\tau} a=1 / T$ are the volume and inverse temperature of the system; $a$ denotes the lattice spacing and $S_{F}$ is the Euclidean action for free fermions of mass $m$.

In the simplest discretization scheme for fermionic actions derivatives are replaced by nearest neighbor differences on a four dimensional lattice,

$$
S_{F}=\sum_{x} \frac{1}{2}\left(\sum_{k=1}^{3}\left(\bar{\psi}_{x} \gamma_{k} \psi_{x+\hat{k}}-\bar{\psi}_{x} \gamma_{k} \psi_{x-\hat{k}}\right)+\mathrm{e}^{\mu a} \bar{\psi}_{x} \gamma_{4} \psi_{x+\hat{4}}-\mathrm{e}^{-\mu a} \bar{\psi}_{x} \gamma_{4} \psi_{x-\hat{4}}\right)+m a \bar{\psi}_{x} \psi_{x}
$$

Here we also introduced the chemical potential through the usual exponential form [13]. The Grassmann-valued fermion fields obey anti-periodic boundary conditions in the time direction. We note that the transformation $\psi_{\vec{x}, x_{4}} \rightarrow$ $\mathrm{e}^{-\mu x_{4}} \psi_{\vec{x}, x_{4}}, \bar{\psi}_{\vec{x}, x_{4}} \rightarrow \mathrm{e}^{\mu x_{4}} \bar{\psi}_{\vec{x}, x_{4}}$ leaves the path integral over the fermion fields invariant and shifts the entire $\mu$ dependence of the fermion action into the last time slice. The Euclidean action thus only depends on the chemical potential in units of the temperature, $\mu / T=\mu a N_{\tau}$. The action can be written in momentum space as ${ }^{1}$

$$
S_{F}=\sum_{p, \nu} \bar{\psi}(p) i \gamma_{\nu} D_{\nu}(p, \mu) \psi(p)
$$

This leads immediately to the denominator of the fermion propagator

$$
D\left(\vec{p}, p_{4}, \mu\right) \equiv \sum_{\nu=1}^{4} D_{\nu}\left(\vec{p}, p_{4}, \mu\right) D_{\nu}\left(\vec{p}, p_{4}, \mu\right)=\sum_{k=1}^{3} \sin ^{2}\left(a p_{k}\right)+\sin ^{2}\left(a p_{4}-i \mu a\right),
$$

and allows to evaluate the partition function explicitly,

$$
Z(V, T, \mu, 0)=\prod_{p} D^{2}\left(\vec{p}, p_{4}, \mu\right) .
$$

Here the momenta take on discrete values, $a p_{k}=2 \pi n_{k} / N_{\sigma}, n_{k}=0,, \pm 1, \ldots, \pm\left(N_{\sigma} / 2-1\right), N_{\sigma} / 2$ and $a p_{4}=2 \pi\left(n_{4}+\right.$ $1 / 2) / N_{\tau}, n_{4}=0,, \pm 1, \ldots, \pm\left(N_{\tau} / 2-1\right),-N_{\tau} / 2$.

For our general discussion of cut-off effects that arise from the finite lattice spacing given in units of the temperature, $a T \equiv 1 / N_{\tau}$, the spatial extent of the lattice is of less interest. We thus take the thermodynamic limit, $N_{\sigma} \rightarrow \infty$,

\footnotetext{
${ }^{1}$ Here and in the following we consider massless fermions, with obvious generalization for the massive case.
} 
which simplifies the following considerations. We then obtain the pressure of a free fermion gas at temperature $T$ and for chemical potential $\mu$,

$$
\begin{aligned}
P a^{4} & =2 \int_{-\pi / 2}^{\pi / 2} \frac{\mathrm{d}^{3} a p}{(2 \pi)^{3}} \frac{1}{N_{\tau}} \sum_{0 \leq a p_{4} \leq \pi} \ln D\left(\vec{p}, p_{4}, \mu\right) \\
& =2 \int_{-\pi / 2}^{\pi / 2} \frac{\mathrm{d}^{3} a p}{(2 \pi)^{3}} \frac{1}{N_{\tau}} \sum_{n=0}^{N_{\tau} / 2-1} \ln \left[\omega^{2}(\vec{p})+\sin ^{2}\left(2 \pi(n+1 / 2) / N_{\tau}-i \mu a\right)\right]
\end{aligned}
$$

with $\omega^{2}(\vec{p})=\sum_{k=1}^{3} \sin ^{2}\left(a p_{k}\right)$. Note that we reduced the integration and summation intervals to half the Brillouin zone which eliminates a factor 16 arising from the 16 so-called doublers which emerge in the naive discretization. We thus normalize to a single quark flavor as we also will do for other discretization schemes in the following sections. In order to evaluate the sum over Matsubara modes appearing in Eq. (7) one uses the fact that this sum can be viewed as the sum over residues resulting from a contour integral over poles in the complex plane [14],

$$
\sum_{n} f\left(r_{n}\right) \rightarrow \oint \frac{d r}{2 \pi i} f(r) h(r, \mu)
$$

The computational steps given in [14] can easily be generalized to non vanishing chemical potential by noting that the entire $\mu$ dependence is contained in the function $h(r, \mu)$,

$$
h(r, \mu)=\frac{e^{\mu a N_{\tau}}}{r\left(r^{N_{\tau}}+e^{\mu a N_{\tau}}\right)},
$$

the poles of which at

$$
r_{n}=\exp \left[i\left(2 \pi(n+1 / 2) / N_{\tau}-i \mu a\right)\right]
$$

deliver the sum over the Matsubara frequencies via the relation

$$
\left.\sin ^{2}\left(2 \pi(n+1 / 2) / N_{\tau}-i \mu a\right)\right)=-\frac{1}{4}\left(r_{n}-\frac{1}{r_{n}}\right)^{2}
$$

Carrying out the sum over the discrete set of momenta yields

$$
\sum_{n=0}^{N_{\tau} / 2-1} \ln \left[\sin ^{2}\left(2 \pi(n+1 / 2) / N_{\tau}-i \mu a\right)+\omega^{2}(\vec{p})\right]=\ln \left(1+z e^{-N_{\tau} a E(\vec{p})}\right)+\ln \left(1+z^{-1} e^{-N_{\tau} a E(\vec{p})}\right)+\text { const. }
$$

where $z=\exp \left(\mu a N_{\tau}\right)=\exp (\mu / T)$ is the fugacity and $E(\vec{p})$ is the dispersion relation which is given by the pole of the fermion propagator, i.e. $E(\vec{p})$ is obtained from the zeroes of Eq.(4) for $\mu a=0$,

$$
D\left(\vec{p}, E=i p_{4}, 0\right)=0 \quad \Leftrightarrow \quad \omega^{2}(\vec{p})-\sinh ^{2}(a E)=0
$$

This emphasizes the particular role dispersion relations play in the study of bulk thermodynamics.

Inserting Eq. (12) into Eq. (7) and subtracting the zero temperature part of the pressure, $\left(P a^{4}\right)_{0} \equiv \lim _{N_{\tau} \rightarrow \infty} P a^{4}$ eliminates ultra-violet divergences and allows to define the pressure in the same normalization as it is used in numerical calculations on the lattice,

$$
\begin{aligned}
\frac{P}{T^{4}} & \equiv\left[P a^{4}-\left(P a^{4}\right)_{0}\right] N_{\tau}^{4} \\
& =\frac{N_{\tau}^{3}}{4 \pi^{3}} \int_{-\pi / 2}^{\pi / 2} \mathrm{~d}^{3} a p\left[\ln \left(1+z \exp \left(-N_{\tau} a E(\vec{p})\right)\right)+\ln \left(1+z^{-1} \exp \left(-N_{\tau} a E(\vec{p})\right)\right)\right] .
\end{aligned}
$$

Eq. (14) is the starting point for a systematic analysis of cut-off effects in bulk thermodynamic observables that are obtained from the logarithm of the partition function, $P / T^{4} \equiv \lim _{V \rightarrow \infty}\left(V T^{3}\right)^{-1} \ln Z$, in terms of derivatives with respect to $T$ or $\mu$. An expansion of $P / T^{4}$ around $1 / N_{\tau}=0$ yields systematic corrections to the continuum ideal gas result which are given in terms of even powers of $1 / N_{\tau}$. This arises after introducing $y_{i}=p_{i} / T=N_{\tau} a p_{i}$ as new integration variable in Eq. (14) and realizing that the dispersion relation is an even function in the momenta. We will discuss this expansion in more detail in the next section. 


\section{IMPROVEMENT OF DISPERSION RELATIONS AND BULK THERMODYNAMICS}

\section{A. Generalized dispersion relations and the pressure}

We will outline here the arguments that lead to the observation that also for more general fermion actions than the one discussed in the previous section there exists a close relation between that branch of the dispersion relation that survives in the continuum limit and the large- $N_{\tau}$ expansion of $P / T^{4}$.

Similarly to the fermion propagator derived in the previous section for the naive discretization scheme of the fermion action one obtains results for general fermion actions of staggered or Wilson type that also may involve more complicated terms than the 1-link term used in the previous section. The propagator for free fermions of staggered type, $D\left(\vec{p}, p_{4}\right)$, can generally be written as a polynomial ${ }^{2}$ in $\sin ^{2}\left(a p_{4}\right)$,

$$
D\left(\vec{p}, p_{4}\right)=\sum_{i=0}^{n} d_{i}(\vec{p}) \sin ^{2 i}\left(a p_{4}\right)
$$

where the coefficients $d_{i}$ depend on the spatial momentum components. For Wilson type quarks the inverse propagator can instead be written as a polynomial in $\sin ^{2}\left(a p_{4} / 2\right)$,

$$
D\left(\vec{p}, p_{4}\right)=\sum_{i=0}^{n} d_{i}(\vec{p}) \sin ^{2 i}\left(a p_{4} / 2\right) .
$$

This is due to the Wilson term which introduces a $\cos \left(a p_{4}\right)=1-2 \sin ^{2}\left(a p_{4} / 2\right)$. In both cases, rewriting $D$ in terms of its $n$ roots, $\omega_{i}(\vec{p})$,

$$
D\left(\vec{p}, k_{4}\right)=d_{n}(\vec{p}) \prod_{i=1}^{n}\left[\sin ^{2}\left(a k_{4}\right)+\omega_{i}^{2}(\vec{p})\right]
$$

where $k_{4}=p_{4}$ for staggered and $k_{4}=p_{4} / 2$ for Wilson quarks, immediately gives the $n$ dispersion relations,

$$
\sinh ^{2}\left(a \epsilon_{i}(\vec{p})\right)=-\sin ^{2}\left(a k_{4}\right)=\omega_{i}^{2}(\vec{p}), i=1, . ., n
$$

with $\epsilon_{i}=E_{i}$ for staggered and $\epsilon_{i}=E_{i} / 2$ for Wilson type quarks. Although $D$ is real, the roots can in general be complex. However, at least for small momenta the root which survives the continuum limit, $\omega_{1}$ to be definite, must be real in order to reproduce the continuum dispersion relation, $E^{2}(\vec{p})=p^{2}$. When we insert the right hand side of Eq. (17) in the partition function, Eq. (5), we find that each factor appearing in the product over the $n$ distinct roots contributes in the evaluation of $P / T^{4}$ as a term in a sum over the different branches, $E_{i}$, of the dispersion relation. Each term in this sum has the same structure as the integral appearing in Eq. (14). In the large- $N_{\tau}$ limit, however, all branches that have a gap in the dispersion relation at $p=0$ give contributions to $P / T^{4}$ that are exponentially suppressed for large- $N_{\tau}$. Also for more general actions Eq. (14) thus is the starting point for the analysis of cut-off effects in the large- $N_{\tau}$ limit. In order to analyze power-like corrections to bulk thermodynamics in the continuum limit it suffices to analyze the properties of $E(\vec{p}) \equiv E_{1}(\vec{p})$.

\section{B. Rotational invariance, improved dispersion relations and improved thermodynamics}

For both, standard staggered and standard Wilson fermions the dispersion relation receives $\mathcal{O}\left(a^{2}\right)$ corrections,

$$
E^{2}(\vec{p})=p^{2}+\mathcal{O}\left(a^{2} p^{4}\right)+\mathcal{O}\left(a^{2} \sum_{k=1}^{3} p_{k}^{4}\right)
$$

with $p^{2}=\vec{p}^{2}=\sum_{k=1}^{3} p_{k}^{2}$. Improving the dispersion relation moves the leading $\mathcal{O}\left(a^{2}\right)$ lattice artefacts to some higher order $n$. For this purpose it suffices to construct an action with rotational invariance maintained to this order. To

\footnotetext{
${ }^{2}$ We give here relations for $\mu a=0$. The chemical potential can, however, be reintroduced at any stage through the substitution $a p_{4} \rightarrow a p_{4}-i \mu a$.
} 
see how this works let us briefly discuss the dispersion relation for standard staggered fermions which is identical to Eq.(13). Expanding this relation for small values of $a p_{k}$ and $a E$ up to terms of order $p_{k}^{4}, E^{4}$ yields

$$
E^{2}-p^{2}+\frac{1}{3} \sum_{k=1}^{3} a^{2} p_{k}^{4}+\frac{1}{3} a^{2} E^{4}=0
$$

which has the solution

$$
E^{2}(\vec{p})=p^{2}-\frac{1}{3} a^{2} p^{4}-\frac{1}{3} \sum_{k=1}^{3} a^{2} p_{k}^{4},
$$

i.e. corrections to the continuum result, $E(p)=p$, start at $\mathcal{O}\left(a^{2}\right)$. In a similar way we may proceed for improved actions which have a Euclidean propagator that is rotationally invariant at $\mathcal{O}\left(p^{4}\right)$, i.e. it still may contain corrections at $\mathcal{O}\left(a^{2}\right)$, however terms proportional to $a^{2} \sum_{\mu} p_{\mu}^{4}$ are absent. The important new feature is that the low momentum expansion of $D$, for the branch $E_{1}$ surviving the continuum limit, now has the form

$$
\left(E_{1}^{2}-p^{2}\right)\left[1+f\left(a p_{k}, a E_{1}\right)\right]+\mathcal{O}\left(a^{4} p_{\mu}^{6}\right)=0
$$

with $f\left(a p_{k}, a E_{1}\right)=$ const $a^{2}\left(E_{1}^{2}-p^{2}\right)$ which leads to

$$
E_{1}^{2}=p^{2}+\mathcal{O}\left(a^{4} p_{\mu}^{6}\right)
$$

In general, if the propagator can be factorized, up to order $a^{n} p_{\mu}^{n+2}$, into a factor $E_{1}^{2}-p^{2}$, with $E_{1}$ corresponding to the lowest root, times 1 plus some function $f\left(a p_{k}, a E_{1}\right)$ which starts at $\mathcal{O}\left(a^{2}\right)$ then

$$
a^{-2} D\left(E_{1}, \vec{p}\right)=\left(E_{1}^{2}-p^{2}+\mathcal{O}\left(a^{n} p_{\mu}^{n+2}\right)\right)\left[1+f\left(a p_{k}, a E_{1}\right)\right]+\mathcal{O}\left(a^{n} p_{\mu}^{n+2}\right)
$$

and the dispersion relation receives $\mathcal{O}\left(p^{n+2}\right)$ corrections only i.e. it is $\mathcal{O}\left(a^{n-2}\right)$ or $\mathcal{O}\left(p^{n}\right)$ improved.

Suppose now that the dispersion relation is $\mathcal{O}\left(a^{n-2}\right)$ improved,

$$
E_{1}^{2}=p^{2}+\mathcal{O}\left(a^{n} p_{\mu}^{n+2}\right)
$$

In this case the corrections to $E_{1} / T$ start at $\mathcal{O}\left(N_{\tau}^{-n}\right)$,

$$
\frac{E_{1}}{T}=N_{\tau} a E_{1}=\frac{p}{T}\left[1+\mathcal{O}\left(\frac{1}{N_{\tau}} \frac{p}{T}\right)^{n}\right]=\frac{p}{T}[1+\Delta],
$$

where $\Delta$ is a polynomial with only even powers in $1 / N_{\tau}$.

We may now use this expansion to evaluate corrections to the continuum result for the pressure. To do so we rearrange the argument of the logarithms in Eq. (14); with $y_{i}=p_{i} / T=N_{\tau} a p_{i}, y=p / T=\sqrt{y_{1}^{2}+y_{2}^{2}+y_{3}^{2}}$ and $\Delta \equiv \Delta\left(y_{1} / N_{\tau}, y_{2} / N_{\tau}, y_{3} / N_{\tau}\right)$ one has

$$
1+A e^{-E_{1} / T}=\left(1+A e^{-y}\right)\left(1-B\left(1-e^{-y \Delta}\right)\right)
$$

where $B$ is given as $B(y, A)=1 /\left(A^{-1} e^{y}+1\right)$ and $A=z$ or $z^{-1}$, respectively. After expanding in $\Delta$ one then finds for the logarithms appearing in Eq. (14)

$$
\ln \left(1+A \exp \left(-N_{\tau} a E(\vec{p})\right)\right)=\ln \left(1+A \mathrm{e}^{-y}\right)-B y \Delta+\mathcal{O}\left(\Delta^{2}\right) .
$$

While the first term will give the continuum ideal gas result for the pressure of a fermion gas, the second term and further higher order corrections will generate a systematic expansion in powers of $1 / N_{\tau}$. This shows that improving the dispersion relation to a certain order in $1 / N_{\tau}$ immediately leads to an improvement of the high temperature limit of free energy, pressure and other thermodynamic observables derived from these to the same order. This also is the case for derivatives of $P / T^{4}$ with respect to the chemical potential, such as the quark number density or higher order susceptibilities. We will expand on this in the next subsection. In the following it also will be convenient to introduce polar coordinates for $y_{i}$, which allows us to think of the cut-off dependence entering only in the radial component, i.e. we take the arguments of $\Delta$ to be $y / N_{\tau}, \phi, \theta$ and rewrite it as

$$
\Delta \equiv \Delta\left(y / N_{\tau}, \phi, \theta\right)=\sum_{k=1}^{\infty} a_{2 k}(\phi, \theta)\left(\frac{y}{N_{\tau}}\right)^{2 k} .
$$

The lowest non-vanishing contribution will appear at $k=n / 2$. 


\section{Cut-off dependence of the pressure}

We are now in a position to discuss the systematic expansion of the pressure in inverse powers of $N_{\tau}$ and analyze systematically cut-off effects and there dependence on temperature $\left(a T=1 / N_{\tau}\right)$ as well as chemical potential $(\mu / T=$ $\mu a N_{\tau}$ ). We insert Eq. (27) into Eq. (14) and obtain

$$
\begin{aligned}
\frac{P}{T^{4}}= & \left(\frac{P}{T^{4}}\right)_{S B} \\
& +\frac{2}{(2 \pi)^{3}} \int_{0}^{\pi N_{\tau}} y^{2} \mathrm{~d} y \int_{0}^{\pi} \sin \theta \mathrm{d} \theta \int_{0}^{2 \pi} \mathrm{d} \phi\left[\ln \left(1-B(y, z)\left(1-\mathrm{e}^{-y \Delta}\right)\right)+\ln \left(1-B\left(y, z^{-1}\right)\left(1-\mathrm{e}^{-y \Delta}\right)\right)\right]
\end{aligned}
$$

where the leading order ideal gas term is given by

$$
\begin{aligned}
\left(\frac{P}{T^{4}}\right)_{S B} & =\frac{1}{\pi^{2}} \int_{0}^{\infty} \mathrm{d} y y^{2}\left[\ln \left(1+z \mathrm{e}^{-y}\right)+\ln \left(1+z^{-1} \mathrm{e}^{-y}\right)\right] \\
& =\frac{4 \pi^{2}}{3} B_{4}\left(\frac{1}{2}\left(1-i \frac{\mu}{\pi T}\right)\right)=\frac{7 \pi^{2}}{180}\left[1+\frac{30}{7}\left(\frac{\mu}{\pi T}\right)^{2}+\frac{15}{7}\left(\frac{\mu}{\pi T}\right)^{4}\right] .
\end{aligned}
$$

Note that the well-known ideal gas result for the pressure is an even Bernoulli polynomial $B_{4}$ of degree 4 in the chemical potential. In the following we will argue that similarly to the leading term also the corrections at order $N_{\tau}^{-2 n}$ are Bernoulli polynomials in $\mu /(\pi T)$ of degree $(4+2 n)$ which might be less obvious.

As the entire cut-off dependence in Eq. (30) arises from the cut-off dependence of the dispersion relation, i.e. from $\Delta=\Delta\left(y / N_{\tau}, \phi, \theta\right)$, we may first expand the pressure in a Taylor series around $y \Delta(0, \phi, \theta)=0$. After noting that derivatives with respect to $y \Delta$ can be replaced by derivatives with respect to $y$ we arrive at

$$
\frac{P}{T^{4}}-\left(\frac{P}{T^{4}}\right)_{S B}=\frac{1}{4 \pi^{3}} \sum_{n=1}^{\infty} \frac{1}{n !} \int_{0}^{\pi N_{\tau}} y^{2} \mathrm{~d} y \int_{0}^{\pi} \sin \theta \mathrm{d} \theta \int_{0}^{2 \pi} \mathrm{d} \phi(y \Delta)^{n}\left(\frac{\partial^{n-1} B(y, z)}{\partial y^{n-1}}+\frac{\partial^{n-1} B\left(y, z^{-1}\right)}{\partial y^{n-1}}\right) .
$$

Using the representation of $\Delta$ given in Eq. (29) we now can rearrange the above series and obtain an expansion in even powers of $1 / N_{\tau}$,

$$
\frac{P}{T^{4}}-\left(\frac{P}{T^{4}}\right)_{S B}=\sum_{k=1}^{\infty} A_{2 k} P_{2 k}\left(\frac{\mu}{\pi T}\right)\left(\frac{\pi}{N_{\tau}}\right)^{2 k},
$$

where $A_{2 k}$ results from the integration over angular variables and the chemical potential dependent part, $P_{2 k}(\mu / \pi T)$, is given in terms of a Bernoulli polynomial that results from the integration over the radial coordinate $y$,

$$
\begin{aligned}
P_{2 k}\left(\frac{\mu}{\pi T}\right) & \equiv \frac{1}{\left(2^{-3-2 k}-1\right) b_{4+2 k}} B_{4+2 k}\left(\frac{1}{2}-i \frac{1}{2} \frac{\mu}{\pi T}\right) \\
& =\sum_{l=0}^{k+2}(-1)^{2+k-l}\left(\begin{array}{c}
4+2 k \\
2 l
\end{array}\right) \frac{2^{2 l-1}-1}{2^{3+2 k}-1} \frac{b_{2 l}}{b_{4+2 k}}\left(\frac{\mu}{\pi T}\right)^{4+2 k-2 l}
\end{aligned}
$$

with $b_{n}$ denoting Bernoulli numbers. The functions $P_{n}$ have been normalized such that $P_{n}(0)=1$. Note that the ideal gas result can similarly be written as $A_{0} P_{0}(\mu / \pi T)$ with $A_{0}=7 \pi^{2} / 180$. We give some more details on the derivation of Eq. (33) and the definition of $A_{2 k}$ in Appendix $\mathrm{A}$ The point we want to stress here is that the dependence of cut-off effects on the chemical potential arises only through a Bernoulli polynomial of degree $4+2 k$. For the particular complex argument appearing in Eq. (34) the Bernoulli polynomial contains only even powers of the chemical potential, reflecting the particle anti-particle symmetry, $\mu \leftrightarrow-\mu$, of the partition functions. We also note that all terms in Eq. (34) are positive. Cut-off effects thus become larger with increasing $\mu / T$, although the effect is small as exact evaluations of the pressure for fixed $N_{\tau}$ show [9].

Eqs. (33) and (34) are the main result of this section. With this we have shown that in the high temperature, ideal gas limit of QCD cut-off effects in the fermion sector of bulk thermodynamic observables can be traced back to the cut-off dependence of the dispersion relation for the quark propagator. The structure of the cut-off dependence is preserved at non-zero chemical potential $\mu$, i.e. actions which are improved to a certain order in $a T \equiv 1 / N_{\tau}$ at $\mu=0$ are so also for $\mu>0$. Moreover, we find that the $\mu$-dependence of cut-off effects is universal, i.e. $\mu$-dependent correction factors at $\mathcal{O}\left(N_{\tau}^{n}\right)$ are independent of the discretization and improvement schemes and are proportional to a Bernoulli polynomial of degree $4+n$.

In the following we give explicit results for cut-off dependent corrections to the continuum ideal gas limit for several fermion actions and compare the asymptotic large $N_{\tau}$ behavior derived in this section with an explicit evaluation of partition functions at small values of $N_{\tau}$. 


\section{STAGGERED TYPE QUARKS}

We now want to apply the general considerations presented in the previous section to the case of staggered fermions. We restrict ourselves to the class of staggered actions which contain terms with fermion and anti-fermion fields separated by up to three links,

$$
S_{F}=\sum_{x, y} \bar{\chi}(x) M(x, y) \chi(y)
$$

with

$$
\begin{aligned}
M(x, y)= & \sum_{\mu=1}^{4} \eta_{\mu}(x)\left(\sum_{i=1,3} c_{i, 0}[\delta(x+i \hat{\mu}, y)-\delta(x-i \hat{\mu}, y)]\right. \\
& \left.+\sum_{\nu \neq \mu} \sum_{j= \pm 2} c_{1, j}[\delta(x+\hat{\mu}+j \hat{\nu}, y)-\delta(x-\hat{\mu}+j \hat{\nu}, y)]\right) .
\end{aligned}
$$

To reproduce the correct continuum limit the expansion coefficients are constrained by the relation

$$
c_{1,0}+3 c_{3,0}+6 c_{1,2}=\frac{1}{2} .
$$

Within this class of staggered fermion actions one can construct actions that are rotationally invariant up to $\mathcal{O}\left(p^{4}\right)$, i.e. the denominator of the fermion propagator, $D(\vec{p})$, becomes a function of only $p^{2}$ up to this order. This can be achieved with the additional constraint [3]

$$
c_{1,0}+27 c_{3,0}+6 c_{1,2}=24 c_{1,2} \stackrel{E q \cdot \underline{37}}{\longrightarrow} c_{1,2}=1 / 48+c_{3,0}
$$

Particularly well-known versions of 3-link actions that are currently used in studies of QCD thermodynamics with staggered fermions are the Naik action [2], for which $c_{1,2} \equiv 0$, and the p4 action [3] where $c_{3,0} \equiv 0$.

For the general three link action, not yet restricted to the subset of rotational invariant propagators, the propagator in momentum space is a polynomial of degree $3 \mathrm{in} \sin ^{2}\left(a p_{4}\right)$, i.e. it is of the generic form given in Eq. (15). Following the discussion presented in the previous section one may work out the dispersion relation on the branch that survives in the continuum limit. For the leading correction to the continuum result one finds

$$
\frac{E_{1}}{p}=1-\frac{1}{6}\left(1-48\left(c_{1,2}-c_{3,0}\right)\right)\left(p^{2}+\frac{1}{p^{2}} \sum_{k=1}^{3} p_{k}^{4}\right) a^{2}+\mathcal{O}\left(a^{4}\right),
$$

where we have used Eq. (37) to eliminate $c_{1,0}$.

As corrections to the continuum dispersion relation, $E_{1}=p$, start at $\mathcal{O}\left(a^{2}\right)$, bulk thermodynamic observables will receive cut-off dependent corrections starting at the same order, $(a T)^{2}=1 / N_{\tau}^{2}$. Eqs. (39) and (38), however, show that all three link actions with a rotationally invariant propagator will lead to an $\mathcal{O}\left(a^{2}\right)$ improved dispersion relation and thus also to $\mathcal{O}\left(a^{2}\right)$ improved bulk thermodynamics. In the class of three link staggered fermion actions one finds for the $\mathcal{O}\left(a^{2}\right)$ improved dispersion relations,

$$
\begin{aligned}
\frac{E_{1}}{p}= & 1+\frac{3}{40}\left\{p^{4}-\frac{1}{p^{2}} \sum_{k=1}^{3} p_{k}^{6}\right\} a^{4} \\
& \left.-\frac{1}{756}\left(1+1344 c_{3,0}\right)\right)\left(p^{3}-\frac{1}{p} \sum_{i<j} p_{i}^{2} p_{j}^{2}\right)^{2} a^{6}+\frac{1}{14}\left(p_{1} p_{2} p_{3}\right)^{2} a^{6}+\mathcal{O}\left(a^{8}\right) .
\end{aligned}
$$

We note that all three link actions lead to identical leading order corrections to the continuum dispersion relation, but differ at subleading order. According to the discussion presented in the previous section, this feature will carry over also to the cut-off dependence of bulk thermodynamics at zero and non-zero chemical potential. 


\begin{tabular}{|c|c|c|c|}
\hline action & $A_{2} / A_{0}$ & $A_{4} / A_{0}$ & $A_{6} / A_{0}$ \\
\hline \hline standard staggered & $248 / 147$ & $635 / 147$ & $3796 / 189$ \\
\hline Naik & 0 & $-1143 / 980$ & $-365 / 77$ \\
\hline p4 & 0 & $-1143 / 980$ & $73 / 2079$ \\
\hline \hline standard Wilson & $248 / 147$ & $635 / 147$ & $13351 / 8316$ \\
\hline hypercube & -0.242381 & 0.114366 & -0.0436614 \\
\hline \hline $\begin{array}{c}\text { overlap/ } \\
\text { domain wall }\end{array}$ & $248 / 147$ & $635 / 147$ & $3796 / 189$ \\
\hline
\end{tabular}

TABLE I: Coefficients in the series expansion (Eq. (33)) for the pressure calculated using different actions. The expansion coefficients have been normalized to the ideal Fermi gas value at vanishing chemical potential, $A_{0} \equiv\left(p / T^{4}\right)_{S B}(\mu / T=0)=$ $7 \pi^{2} / 180$.

We have evaluated the leading cut-off dependent corrections to the continuum ideal gas pressure for a generic 3-link, staggered fermion action up to $\mathcal{O}\left(1 / N_{\tau}^{6}\right)$ using Mathematica to perform the expansion of the integrand appearing in Eq. (30) in inverse powers of $N_{\tau}$. For the pressure we find,

$$
\begin{aligned}
\frac{180}{7 \pi^{2}}\left[\frac{P}{T^{4}}-\left(\frac{P}{T^{4}}\right)_{S B}\right]= & \frac{248\left(1-48\left(c_{1,2}-c_{3,0}\right)\right)}{147} P_{2}\left(\frac{\mu}{\pi T}\right)\left(\frac{\pi}{N_{\tau}}\right)^{2} \\
& +\left(\frac{635}{147}+\frac{16256\left(c_{3,0}-c_{1,2}\right)}{35}+\frac{2365248\left(c_{3,0}-c_{1,2}\right)^{2}}{245}\right) P_{4}\left(\frac{\mu}{\pi T}\right)\left(\frac{\pi}{N_{\tau}}\right)^{4} \\
& +\frac{73}{2079}\left(1+6528 c_{3,0}+\left(1-48\left(c_{1,2}-c_{3,0}\right)\right)\left(571+1018368 c_{1,2}^{2}\right.\right. \\
& \left.\left.+51888 c_{3,0}+1331712 c_{3,0}^{2}-48 c_{1,2}\left(1217+48960 c_{3,0}\right)\right)\right) P_{6}\left(\frac{\mu}{\pi T}\right)\left(\frac{\pi}{N_{\tau}}\right)^{6} \\
& +\mathcal{O}\left(\left(\pi / N_{\tau}\right)^{8}\right),
\end{aligned}
$$

with $P_{n}$ defined in Eq. (34). Results for the standard staggered (1-link) action as well as for the class of $\mathcal{O}\left(a^{2}\right)$ improved 3-link actions, for which $c_{1,2}=1 / 48+c_{3,0}$, are easily read off from Eq. (41). In Table [ we give the expansion coefficients for the standard staggered action as well as the Naik and p4 actions. We note that coefficients of the sub-leading corrections to the pressure calculated with the standard staggered action as well as with the Naik action are larger than the leading expansion coefficient. The subleading correction for the Naik action is a factor 137 larger than for the p4-action.

It is instructive to compare the asymptotic large $N_{\tau}$ behavior of the cut-off dependent corrections to the continuum ideal Fermi gas with the exact results for the pressure evaluated at fixed values of $N_{\tau}$ by using Eq. (6). At vanishing chemical potential this is shown for standard staggered, Naik and p4 improved fermions in Fig [1. Here we have normalized the exact lattice results to the continuum Stefan-Boltzmann limit, $7 \pi^{2} / 180$. We note that the exact calculation of the partition function at fixed values of $N_{\tau}$ reproduces the basic pattern derived from the leading orders of the large- $N_{\tau}$ expansion. Cut-off effects in the pressure are reduced when using improved discretization schemes.

\section{WILSON TYPE QUARKS}

We will now consider the case of Wilson fermions. Since in the interacting case cut-off effects arise in the Wilson fermion formulation already at $\mathcal{O}\left(a g^{2}\right)$ improvement schemes developed for Wilson fermions have primarily focused on removing terms linear in the cut-off (clover improved actions [4]). Actions that are $\mathcal{O}\left(a^{2} g^{0}\right)$ improved received much less attention and, in fact, are currently not exploited in studies of QCD thermodynamics with Wilson fermions. Nonetheless, removing or at least reducing $\mathcal{O}\left(a^{2}\right)$ effects in the Wilson fermion formulations is as important as in the staggered case when one wants to perform studies of QCD thermodynamics at high temperature within this discretization scheme. We will discuss here a generic Wilson type action with couplings constrained to a hypercube of size $(2 a)^{4}$. This type of action is discussed in the context of truncated fixed point actions 6, 15]. Some exploratory studies of thermodynamics with dynamical hypercube fermions have been presented in [16]. The Dirac matrix for 

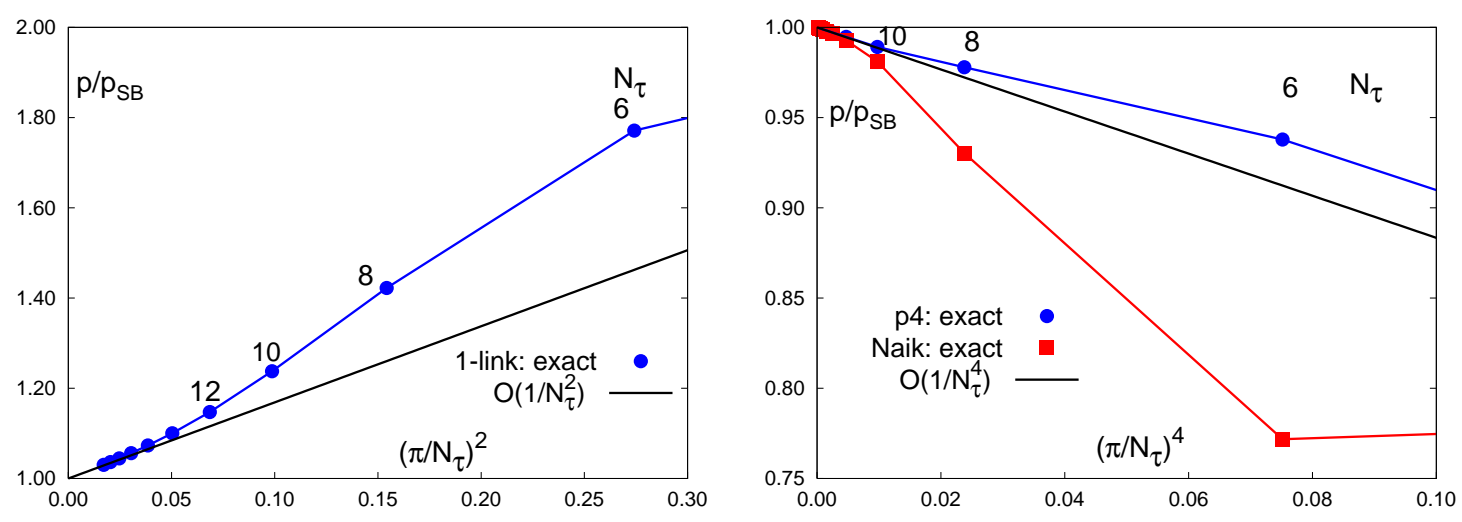

FIG. 1: The pressure for staggered type quarks normalized to the continuum Stefan Boltzmann limit. The left hand figure shows results for the (standard) 1-link action and the left hand figure gives results for the Naik and p4 actions. In both cases we compare exact results, calculated on lattices with temporal extent $N_{\tau}$ and infinite spatial extent, with the leading $1 / N_{\tau}^{n}$ correction. Note the different vertical scales.

this generic Wilson action can be written as

$$
M(x, y)=\sum_{\mu=1}^{4} \gamma_{\mu} \rho_{\mu}(x-y)+\lambda(x-y)
$$

with

$$
\begin{aligned}
\rho_{\mu}(x-y)= & \rho_{1}[\delta(y, x+\hat{\mu})-\delta(y, x-\hat{\mu})] \\
& +\rho_{2} \sum_{\hat{\nu}}[\delta(y, x+\hat{\mu}+\hat{\nu})-\delta(y, x-\hat{\mu}+\hat{\nu})] \\
& +\rho_{3} \sum_{\hat{\nu}, \hat{\rho}}[\delta(y, x+\hat{\mu}+\hat{\nu}+\hat{\rho})-\delta(y, x-\hat{\mu}+\hat{\nu}+\hat{\rho})] \\
& +\rho_{4} \sum_{\hat{\nu}, \hat{\rho}, \hat{\sigma}}[\delta(y, x+\hat{\mu}+\hat{\nu}+\hat{\rho}+\hat{\sigma})-\delta(y, x-\hat{\mu}+\hat{\nu}+\hat{\rho}+\hat{\sigma})]
\end{aligned}
$$

for the vector terms and

$$
\begin{aligned}
\lambda(x-y)= & \lambda_{0} \delta(y, x) \\
& +\lambda_{1} \sum_{\hat{\mu}}[\delta(y, x+\hat{\mu})+\delta(y, x-\hat{\mu})] \\
& +\lambda_{2} \sum_{\hat{\mu}, \hat{\nu}}[\delta(y, x+\hat{\mu}+\hat{\nu})+\delta(y, x-\hat{\mu}+\hat{\nu})] \\
& +\lambda_{3} \sum_{\hat{\mu}, \hat{\nu}, \hat{\rho}}[\delta(y, x+\hat{\mu}+\hat{\nu}+\hat{\rho})+\delta(y, x-\hat{\mu}+\hat{\nu}+\hat{\rho})] \\
& +\lambda_{4} \sum_{\hat{\mu}, \hat{\nu}, \hat{\rho}, \hat{\sigma}}[\delta(y, x+\hat{\mu}+\hat{\nu}+\hat{\rho}+\hat{\sigma})+\delta(y, x-\hat{\mu}+\hat{\nu}+\hat{\rho}+\hat{\sigma})]
\end{aligned}
$$

for the scalar ones. The sums over $\hat{\nu}, \hat{\rho}, \hat{\sigma}$ extend over positive and negative directions and are mutually orthogonal to each other and to $\hat{\mu}$. Examples for this type of action are the standard Wilson action (including the clover improved version of it) and the hypercube truncated perfect action [6, 15], with coefficients as listed in Table [1] for the massless case.

The denominator of the fermion propagator, $D(p)$, is most conveniently written as

$$
D\left(\vec{p}, p_{4}\right)=R(\vec{p})+2 P(\vec{p}) \cos \left(a p_{4}\right)+Q(\vec{p}) \cos ^{2}\left(a p_{4}\right) .
$$

Note that this complies with Eq.(16) since $\cos \left(a p_{4}\right)$ is to be rewritten as $1-2 \sin ^{2}\left(a p_{4} / 2\right)$. The coefficients $P, Q$ and $R$ depend on $\vec{p}$ and are listed in Appendix B, The requirement that in the limit of $a p_{\mu} \rightarrow 0$ for all $\mu=1, . ., 4$ the 


\begin{tabular}{|c|c|c||c|c|c|}
\hline & Hypercube & Wilson & & Hypercube & Wilson \\
\hline \hline & & & $\lambda_{0}$ & 1.852720547 & 4 \\
$\rho_{1}$ & 0.136846794 & $1 / 2$ & $\lambda_{1}$ & -0.060757866 & $-1 / 2$ \\
$\rho_{2}$ & 0.032077284 & 0 & $\lambda_{2}$ & -0.030036032 & 0 \\
$\rho_{3}$ & 0.011058131 & 0 & $\lambda_{3}$ & -0.015967620 & 0 \\
$\rho_{4}$ & 0.004748991 & 0 & $\lambda_{4}$ & -0.008426812 & 0 \\
\hline
\end{tabular}

TABLE II: Coefficients $\rho_{i}$ and $\lambda_{i}$ for standard Wilson quarks and for the hypercube action.

continuum dispersion relation is obtained leads to the constraints (see also [17])

$$
\begin{aligned}
2 \rho_{1}+12 \rho_{2}+24 \rho_{3}+16 \rho_{4} & =1, \\
\lambda_{0}+8 \lambda_{1}+24 \lambda_{2}+32 \lambda_{3}+16 \lambda_{4} & =0 .
\end{aligned}
$$

Moreover, demanding that the so-called doublers receive a non-vanishing mass at the corners of the Brillouin zone, $a p_{\mu}=\pi$ for one or more component $\mu$, introduces further conditions to be satisfied by the $\lambda$ coefficients,

$$
\begin{aligned}
\lambda_{0}+4 \lambda_{1}-16 \lambda_{3}-16 \lambda_{4} & \neq 0 \\
\lambda_{0}-8 \lambda_{2}+16 \lambda_{4} & \neq 0 \\
\lambda_{0}-4 \lambda_{1}+16 \lambda_{3}-16 \lambda_{4} & \neq 0 \\
\lambda_{0}-8 \lambda_{1}+24 \lambda_{2}-32 \lambda_{3}+16 \lambda_{4} & \neq 0
\end{aligned}
$$

In the standard Wilson action these conditions are realized such that the so-called doubler masses are $2 / a, 4 / a, 6 / a$ and $8 / a$ while for hypercube fermions these masses are $\simeq 2 / a$ with deviations of at most 2 per cent for all corners ${ }^{3}$.

We may now go through the steps outlined in Section II to determine the cut-off dependence of dispersion relations and bulk thermodynamics. We start by expanding the quark propagator, Eq. (45), for small momenta, taking into account the constraints given in Eq. (46),

$$
D\left(\vec{p}, p_{4}\right)=(p a)^{2}-\frac{1}{3}\left(-1+12 \rho_{2}+48 \rho_{3}+48 \rho_{4}\right)\left((p a)^{4}-\sum_{i=1}^{4}\left(a p_{i}\right)^{4}\right)+\mathcal{O}\left(a^{6}\right) .
$$

With the couplings in the hypercube action as listed in Table I the propagator receives $\mathcal{O}\left(a^{2}\right)$ corrections, which however are small when compared to the standard 1-link Wilson action. For the latter the prefactor in Eq. (48) equals $1 / 3$ while for the hypercube action it is -0.0479 . Of course, with an appropriate choice of the couplings $\rho_{2}, \rho_{3}$ and $\rho_{4}$ one can also eliminate the $\mathcal{O}\left(a^{2}\right)$ corrections to the propagator of the hypercube Wilson action completely, making it rotational invariant up to that order.

Turning now to the dispersion relation, from Eq.(45) one obtains, for the branch relevant to the continuum limit,

$$
\sinh ^{2}\left(\frac{a E_{1}}{2}\right)=-\frac{1}{2}\left(1+\frac{P+\sqrt{P^{2}-Q R}}{Q}\right)
$$

The low momentum expansion of this dispersion relation yields,

$$
\frac{E_{1}}{p}=1+\frac{1}{6}\left(-1+12 \rho_{2}+48 \rho_{3}+48 \rho_{4}\right)\left(p^{2}+\frac{1}{p^{2}} \sum_{k=1}^{3} p_{k}^{4}\right) a^{2}+\mathcal{O}\left(a^{4}\right) .
$$

We note that for standard Wilson fermions $\left(\rho_{2}=\rho_{3}=\rho_{4}=0\right)$ the leading order correction to the continuum dispersion relation is identical to that of staggered fermions. This will, of course, carry over also to the leading corrections to bulk thermodynamic observables. Moreover, a choice $-1+12 \rho_{2}+48 \rho_{3}+48 \rho_{4}=0$ not only removes the rotational symmetry breaking term, $\sim \sum_{k=1}^{3} p_{k}^{4}$, in the propagator and the dispersion relation, but eliminates the $\mathcal{O}\left(a^{2}\right)$ corrections completely.

3 Note that with five equations the $\lambda$ coefficients can be determined uniquely once the doubler masses have been fixed. Indeed, fixing these masses to $2 / a$ leads to the HF scalar couplings within 2 per cent accuracy. 

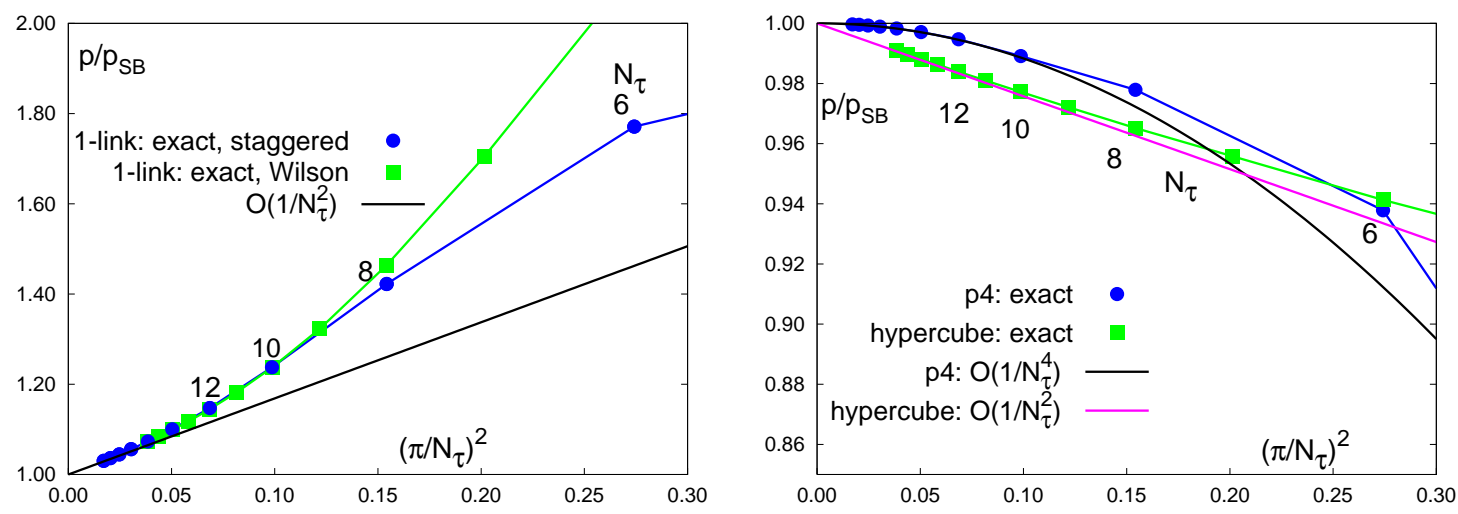

FIG. 2: Pressure for standard Wilson fermions (left) and hypercube Wilson fermions (right) normalized to the continuum Stefan Boltzmann result. In the left hand part we compare the results obtained with standard Wilson fermions to that of standard staggered fermions. Both discretization schemes have identical cut-off errors in leading order $1 / N_{\tau}^{2}$. The right hand part compares results for the hypercube action with the $\mathrm{p} 4$ action.

Using the dispersion relation for the hypercube action, Eq. (49), in our basic relation for the pressure, Eq. (30), we again can perform a systematic expansion for large $N_{\tau}$. With this we obtain for the leading cut-off corrections to the continuum pressure,

$$
\begin{aligned}
\frac{180}{7 \pi^{2}}\left[\frac{P}{T^{4}}-\left(\frac{P}{T^{4}}\right)_{S B}\right]= & -\frac{248}{147}\left(-1+12 \rho_{2}+48 \rho_{3}+48 \rho_{4}\right) P_{2}\left(\frac{\mu}{\pi T}\right)\left(\frac{\pi}{N_{\tau}}\right)^{2} \\
& +\frac{127}{735}\left(25+3492 \rho_{2}^{2}+55872 \rho_{3}^{2}-2148 \rho_{4}+55872 \rho_{4}^{2}\right. \\
& \left.+2328 \rho_{3}\left(-1+48 \rho_{4}\right)+3 \rho_{2}\left(-209+9312 \rho_{3}+9312 \rho_{4}\right)\right) P_{4}\left(\frac{\mu}{\pi T}\right)\left(\frac{\pi}{N_{\tau}}\right)^{4} \\
& +h_{6} P_{6}\left(\frac{\mu}{\pi T}\right)\left(\frac{\pi}{N_{\tau}}\right)^{6}+\mathcal{O}\left(\left(\pi / N_{\tau}\right)^{8}\right) .
\end{aligned}
$$

The explicit form of $h_{6}$ is rather lengthy. It is given in Appendix C

In Fig. 2 we compare exact results for the pressure obtained with standard Wilson fermions and the hypercube action with the leading order large- $N_{\tau}$ expansion obtained in this section. As can be seen in Fig. 2(left) the standard Wilson action and standard staggered fermions have identical leading order cut-off errors and consequently follow an identical pattern for large $N_{\tau}$, e.g. for $N_{\tau} \gtrsim 10$. Cut-off effects for the hypercube action are significantly reduced and of similar magnitude as for the improved staggered actions (Fig. 2(right)). Cut-off dependent corrections at small values of $N_{\tau}$ are already close to the result obtained from the leading order expansion reflecting that higher order corrections are small. This also is apparent from the higher order corrections listed in Table [1.

\section{OVERLAP AND DOMAIN WALL FERMIONS}

Let us now comment on bulk thermodynamics with chirally invariant fermion formulations, e.g. overlap [18] and domain wall fermions [19, 20]. The free field limit of chiral fermion formulations 21] has recently been used to analyze the cut-off dependence of spectral functions at high temperature [22]. We follow this discussion here closely to explore the cut-off dependence of bulk thermodynamics in lattice formulations that use chiral fermion formulations, e.g. overlap or domain wall fermions with standard Wilson kernel.

It is known that in the free field limit the dispersion relations for overlap and domain wall fermions are given by the standard (staggered) dispersion relation up to a constraint, which only alters the large momentum part of the dispersion relations [21]. As we have seen in the previous sections this part of the spectrum does not influence the large$N_{\tau}$ expansion of thermodynamic quantities. Following the general discussion of cut-off effects given in Section II we thus expect that the $1 / N_{\tau}$ expansion for the cut-off dependence of the pressure and related thermodynamic quantities is identical to that of standard staggered fermions. As outlined in Section II this also includes the dependence on a non-zero chemical potential. Nonetheless, in particular for the case of overlap fermions the question has been raised whether the fact that one has to take a square root of e.g. the Wilson fermion operator may lead to difficulties in 
reproducing the correct continuum thermodynamics at non-zero chemical potential [12]. Indeed, the presence of a square root in the basic relation for the partition function (see Eq. (57)) leads to cuts which so far did not show up in our general discussion of bulk thermodynamics in the ideal gas limit. We will, however, show that the cut contributions are exponentially suppressed and do not contribute to the $1 / N_{\tau}$ expansion of the pressure.

We outline here the basic steps that allow to relate also in the overlap formalism the basic properties of the dispersion relation with the cut-off dependence of the pressure. Some more technical considerations are given in Appendix D.

We work with the following form of the (massless) overlap operator [21, 22],

$$
D=\frac{1}{a}\left(1+\frac{X}{\sqrt{X^{\dagger} X}}\right) \text {. }
$$

Here $X=D_{W}-1$, where $D_{W}$ is a nonchiral, undoubled fermion operator like the standard Wilson operator discussed in the previous section. In momentum space $X\left(\vec{p}, p_{4}\right)$ takes on the form

$$
X\left(\vec{p}, p_{4}\right)==\frac{1}{a}\left(i \sum_{\nu=1}^{4} \gamma_{\nu} \sin \left(a p_{\nu}\right)+2 \sum_{\nu=1}^{4} \sin ^{2}\left(a p_{\nu} / 2\right)-1\right) .
$$

In order to keep the notation simple we do not explicitly introduce a chemical potential. It can, however, at any step be re-introduced through the replacement $a p_{4} \rightarrow a p_{4}-i \mu a$.

The fermion propagator of the overlap operator is then given by

$$
D\left(\vec{p}, p_{4}\right)=\frac{1}{2}\left(1-\frac{\sum_{\nu=1}^{4} i \gamma_{\nu} \sin \left(a p_{\nu}\right)}{\sqrt{S^{2}+A^{2}}-A}\right)
$$

where

$$
A \equiv A\left(\vec{p}, p_{4}\right)=1-2 \sum_{\nu=1}^{4} \sin ^{2}\left(a p_{\nu} / 2\right) \quad S^{2} \equiv S^{2}\left(\vec{p}, p_{4}\right)=\sum_{\nu=1}^{4} \sin ^{2}\left(a p_{\nu}\right) .
$$

From Eq. (54) we see that the poles of the fermion propagator are given by

$$
\sqrt{A^{2}+S^{2}}-A=0 \quad \Leftrightarrow \quad S^{2}=0 \text { and } A>0 .
$$

Thus, overlap fermions have indeed exactly the same dispersion relation as naive fermions upto a constraint, $A>0$. The constraint is automatically satisfied at small momenta but has no solutions near the edge of the Brillouin zone, thus avoiding the doubling problem. As pointed out in [22] this carries over to similar relations for the propagator and the poles of free domain wall fermions in the limit of infinite 5th dimension.

With this we obtain for the pressure

$$
P a^{4}=\frac{2}{(2 \pi)^{3}} \int_{-\pi}^{\pi} \mathrm{d}^{3} a p \frac{1}{N_{\tau}} \sum_{a p_{4}} \ln \left(1-\frac{A}{\sqrt{A^{2}+S^{2}}}\right) .
$$

In Eq.(57) the appearance of a square root, of course, does not create any problems; its argument is positive and the pressure thus can be evaluated for any fixed temporal lattice extent $N_{\tau}$. The discussion of the large- $N_{\tau}$ expansion, however, becomes somewhat more complicated than in the cases considered in the previous sections. We also note that we did not reduce the integration range, as it has been done for naive fermions to eliminate contributions from doublers. Integrals and momentum sums still extend over the whole Brillouin zone. Let us denote $x \equiv \sin \left(a p_{4} / 2\right)$. The expression for the pressure may be shown to take the form

$$
P a^{4}=\frac{2}{(2 \pi)^{3}} \int_{-\pi}^{\pi} \mathrm{d}^{3} a p \frac{1}{N_{\tau}} \sum_{a p_{4}} \ln \left(1+\frac{1}{\sqrt{2}} \frac{x^{2}+c^{2}-1 / 2}{\sqrt{c^{2} x^{2}+b^{2}}}\right),
$$

where

$$
\begin{aligned}
b^{2} & =\sum_{i<j} \sin ^{2} a p_{i} / 2 \sin ^{2} a p_{j} / 2+1 / 8=\frac{1}{8}\left(1+\omega^{2}+4\left(c^{4}-c^{2}\right)\right) \\
c^{2} & =\sum_{i=1}^{3} \sin ^{2} a p_{i} / 2, \omega^{2}=\sum_{i=1}^{3} \sin ^{2} a p_{i}
\end{aligned}
$$


The argument of the logarithm may be written as a quadratic form in $\xi=\sqrt{c^{2} x^{2}+b^{2}}$, given by $\left(\xi^{2}+c^{2} \sqrt{2} \xi+\left(c^{2}\left(c^{2}-1 / 2\right)-b^{2}\right)\right) /\left(\sqrt{2} c^{2} \xi\right)$. Hence the numerator may be factorized to obtain

$$
P a^{4}=\frac{2}{(2 \pi)^{3}} \int_{-\pi}^{\pi} \mathrm{d}^{3} a p \frac{1}{N_{\tau}} \sum_{a p_{4}}\left(\ln \left(\sqrt{c^{2} x^{2}+b^{2}}-\xi^{+}\right)+\ln \left(\sqrt{c^{2} x^{2}+b^{2}}-\xi^{-}\right)-\frac{1}{2} \ln \left(c^{2} x^{2}+b^{2}\right)-\ln \left(\sqrt{2 c^{4}}\right)\right),
$$

with $\xi^{ \pm}=-c^{2} / \sqrt{2} \pm \sqrt{b^{2}-\left(c^{4}-c^{2}\right) / 2}=-c^{2} / \sqrt{2} \pm \sqrt{\left(1+\omega^{2}\right) / 8}$. The last term in Eq. (60) is easily identified as a vacuum term that gets canceled once we subtract the zero temperature contribution to the pressure. The third term has a structure similar to that discussed for staggered fermions. It corresponds to a dispersion relation that approaches a non-zero value for small momenta and thus will not contribute to a $1 / N_{\tau}$ expansion for the pressure, i.e. it only leads to exponentially small contributions. We thus have to understand the $N_{\tau}$ dependence of the first two terms. To do so we first carry out the sum over $p_{4}$ which can be done in close analogy to the cases discussed above by first considering the sums

$$
\mathcal{S}^{ \pm}=\frac{\mathrm{d}}{\mathrm{d} \xi^{ \pm}} \frac{1}{N_{\tau}} \sum_{a p_{4}} \ln \left(\sqrt{c^{2} x^{2}+b^{2}}-\xi^{ \pm}\right)=-\frac{1}{N_{\tau}} \sum_{a p_{4}} \frac{1}{\sqrt{c^{2} x^{2}+b^{2}}-\xi^{ \pm}} .
$$

This sum can be evaluated using the same contour integral technique 14 exploited in the case of staggered and Wilson fermions. It, however, needs a bit more care as through the replacement of $x^{2}=\sin ^{2}\left(a p_{4} / 2\right)$ by a complex variable, $x^{2}=-(z-1 / z)^{2} / 4$, the square root in Eq. (61) will have cuts. These contribute to the contour integral. Additional residues appear when doing the sum for $\xi^{+}$as poles appear in Eq. (61) for small momenta when $\xi^{+}>0$. We discuss the calculation of $\mathcal{S}^{ \pm}$in Appendix $\square$ and show there that contributions arising from the cuts differ from a vacuum contribution only by exponentially small terms and thus do not contribute to the large- $N_{\tau}$ expansion of the pressure. This expansion thus receives contributions only from the first term in Eq. (61) and only from that part of the momentum integral where $\xi^{+}>0$. We thus find,

$$
\begin{aligned}
\frac{P}{T^{4}} \equiv\left[P a^{4}-\left(P a^{4}\right)_{0}\right] N_{\tau}^{4}= & \frac{2 N_{\tau}^{3}}{(2 \pi)^{3}} \int_{d(p)} \mathrm{d}^{3} a p \sum_{a p_{4}} \ln \left(\sqrt{c^{2} \sin ^{2}\left(a p_{4} / 2\right)+b^{2}}-\xi^{+}\right) \\
& -\frac{2 N_{\tau}^{4}}{(2 \pi)^{4}} \int_{d(p)} \mathrm{d}^{4} a p \ln \left(\sqrt{c^{2} \sin ^{2}\left(a p_{4} / 2\right)+b^{2}}-\xi^{+}\right)+\mathcal{O}\left(\mathrm{e}^{-\alpha N_{\tau}}\right),
\end{aligned}
$$

where $d(p)$ denotes the section of the 3-d Brillouin zone, in which $\xi^{+}>0$, i.e. for which $1+\omega^{2}>4 c^{4}$ holds. As discussed in Appendix D this result also holds for non-zero chemical potential, with the replacement $a p_{4} \rightarrow a p_{4}-i \mu a$.

We are thus left with an expression very similar to what we have discussed in the previous sections and can express the pressure for overlap fermions through the poles of the denominator appearing in Eq. (61),

$$
\sinh ^{2}\left(E^{+} / 2\right) \equiv \frac{1}{4}\left(z_{i}^{+}-\frac{1}{z_{i}^{+}}\right)^{2}=\frac{b^{2}-\left(\xi^{+}\right)^{2}}{c^{2}}=-\frac{1}{2}\left(1-\sqrt{1+\omega^{2}}\right) \Leftrightarrow \sinh ^{2} E^{+}=\omega^{2}
$$

We now can integrate Eq. (61) to arrive at explicit expressions for Matsubara sums of logarithms. After subtracting again the zero temperature contribution to the pressure one then obtains for the pressure in the overlap formalism an expression that agrees with the corresponding result for staggered fermions, Eq. (14) in a limited region of the Brillouin zone that includes $\vec{p}=0$. Aside from this the pressure for overlap fermions receives only contributions that are exponentially small in $N_{\tau}$. This confirms that the large- $N_{\tau}$ expansion for the pressure in the overlap formulation agrees with that of staggered fermions. As indicated at the beginning of this section this could have been anticipated, for we know that the two actions have the same dispersion relation at small momenta.

For fixed values of $N_{\tau}$ the exact expression for the pressure, Eq. (57), of course, differs from that of naive staggered fermions and cut-off effects thus will be different. Using Eq. (57) we have evaluated the pressure for small values of $N_{\tau}$ explicitly. The results are compared to corresponding calculations performed for standard staggered and Wilson fermions as well as to the common leading order large- $N_{\tau}$ expansion result in Fig. 3(left). We note that the cutoff dependence introduced by the overlap operator for small values of $N_{\tau}$ is smaller than in the staggered fermion discretization scheme. In fact, it is remarkable that already for $N_{\tau}=8$ the exact result for overlap fermions is close to the leading order result of the large- $N_{\tau}$ expansion. For $N_{\tau} \gtrsim 12$ all 1-link discretization schemes yield very similar results and are in good agreement with the leading order large- $N_{\tau}$ expansion.

We finally comment on the ideal gas limit for domain wall fermions. This has previously been analyzed numerically on finite space-time lattices [23]. In the analysis of the cutoff dependence of bulk thermodynamics with domain wall fermions an additional complication arises. One has to introduce additional degrees of freedom, Pauli-Villar 

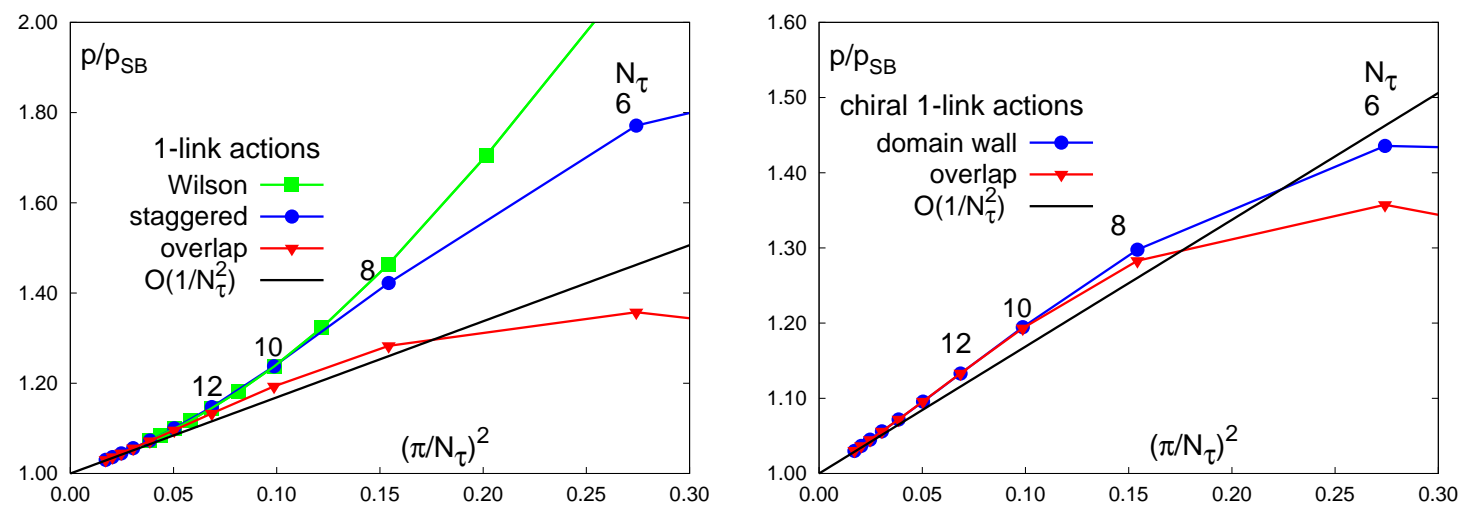

FIG. 3: Pressure for overlap fermions based on the application of the Ginsparg-Wilson operator on a standard Wilson operator (left) and for the domain wall operator using the same kernel with domain wall height $M_{5}=1$ (right). The large- $N_{\tau}$ expansion for both discretization schemes coincides with that of the standard staggered action. In both figures we compare with the leading order $1 / N_{\tau}^{2}$ result.

regulators, which eliminate divergences that arise from the infinite number of heavy fermion fields present in the limit of infinite 5th dimension 24]. The calculation of the free fermion partition function for domain wall fermions, i.e. the free fermion determinant, thus is somewhat more involved [25]. Nonetheless, after diagonalization in momentum space the regularized domain wall fermion determinant takes on a simple form in the limit of infinite extent of the 5th dimension. For massless fermions one obtains,

$$
\operatorname{det} D_{D W}=\prod_{\vec{p}, p_{4}} \mathcal{N}\left(\vec{p}, p_{4}\right)
$$

with

$$
\mathcal{N}\left(\vec{p}, p_{4}\right)=2\left(1+\frac{S^{2}+W^{2}-1}{\sqrt{\left(1+S^{2}+W^{2}\right)^{2}-4 W^{2}}}\right) .
$$

Here $S^{2}$ has been defined in Eq. (55) and $W=1-M_{5}-2 \sum_{\nu=1}^{4} \sin ^{2}\left(a p_{\nu} / 2\right)$ for domain wall height $M_{5}$.

As mentioned before also domain wall fermions have the same dispersion relation as staggered fermions, up to a constraint, which cuts off the spectrum for large momenta. The large- $N_{\tau}$ expansion of bulk thermodynamics in the ideal gas limit thus again will be identical to that of naive staggered fermions. Moreover, for vanishing lattice spacing in the 5th dimension and infinite extent in this direction the domain wall Dirac operator is identical to the overlap operator. It thus may not be surprising that the cut-off effects in the domain wall and in the overlap formalism are quite similar in the free field limit. Differences arise because the sign function is introduced in the domain wall formalism through a discrete set of additional fermion fields [24]. We limit our discussion of cutoff effects in thermodynamics with domain wall fermions here to a comparison of the exact evaluation of the pressure in the domain wall and overlap discretization schemes. With Eqs. (64) and (65) the pressure of free massless domain wall fermions is obtained as

$$
\frac{P}{T^{4}} \equiv\left[P a^{4}-\left(P a^{4}\right)_{0}\right] N_{\tau}^{4}=\frac{2 N_{\tau}^{3}}{(2 \pi)^{3}} \int_{-\pi}^{\pi} \mathrm{d}^{3} a p \sum_{a p_{4}} \ln \mathcal{N}\left(\vec{p}, p_{4}\right)-\frac{2 N_{\tau}^{4}}{(2 \pi)^{4}} \int_{-\pi}^{\pi} \mathrm{d}^{4} a p \ln \mathcal{N}\left(\vec{p}, p_{4}\right) .
$$

In Fig. 3 (right) we show results from a direct evaluation of this expression for some values of $N_{\tau}$ and for domain wall height $M_{5}=1$, which corresponds to the case discussed above for overlap fermions. As can be seen cutoff effects differ for small values of $N_{\tau}$ but rapidly become similar for $N_{\tau} \simeq 8$.

\section{CONCLUSIONS}

We have analyzed here cut-off effects that arise in lattice calculations of bulk thermodynamic observables at high temperature and vanishing as well as non-vanishing chemical potential. We have shown that in the high temperature, ideal gas limit of QCD cut-off effects in fermionic observables can be traced back to the cut-off dependence of the 
dispersion relation for the quark propagator. The structure of the cut-off dependence is preserved at non-zero chemical potential $\mu$, i.e. actions which are improved to a certain order in $a T \equiv 1 / N_{\tau}$ at $\mu=0$ are so also for $\mu>0$. We could show that the $\mu$-dependence of cut-off effects is universal, i.e. $\mu$-dependent correction factors at $\mathcal{O}\left(N_{\tau}^{n}\right)$ are independent of the discretization and improvement schemes. They are given in terms of a Bernoulli polynomial of degree $4+n$; cut-off corrections at order $1 / N_{\tau}^{n}$ depend on an even polynomial in $\mu / \pi T$ of degree $4+n$ with positive coefficients only.

We have shown that the considerations on cut-off effects in thermodynamics with staggered and Wilson fermions carry over to chiral fermion formulations. So far the latter have been used mainly with standard Wilson fermion kernels. The discussion presented in this paper suggest that studies of bulk thermodynamics performed with chiral fermions could profit a lot from the application of improvements schemes similar to those used in studies of bulk thermodynamics with staggered fermions. This certainly can be achieved using the concepts that have been developed to improve standard staggered and Wilson fermion action [26].

\section{Acknowledgments}

We thank Urs Heller for helpful discussions. SS has been supported by the DFG grant GRK/881 and by the EU under the contract no. RII3-CT-2004-506078. FK and EL acknowledge partial support through a grant of the BMBF under contract no. 06BI106. The work of FK and PH has been supported by a contract DE-AC02-98CH10886 with the U.S. Department of Energy.

\section{APPENDIX A: LARGE $N_{\tau}$ EXPANSION OF THE PRESSURE}

We give here some further details on the derivation of the series expansion of the pressure, Eq (33), and give explicit formulas for the expansion coefficients $A_{n}$ as well as the polynomials $P_{n}$ introduced in Eq.(33).

In order to derive Eq. (32) from Eq. (30) as the result of a Taylor expansion $y \Delta$ it is helpful to note that

$$
\frac{\partial \ln \left[1-B(y, z)\left(1-\mathrm{e}^{-y \Delta}\right)\right]}{\partial y \Delta}=\frac{-1}{z \exp (y) \exp (y \Delta)+1} .
$$

It then is obvious that taking further partial derivatives of this logarithm with respect to $y \Delta$ at $y \Delta=0$ is equivalent to taking derivatives with respect to $y$ at $y \Delta=0$. This yields Eq. (32).

The series representation of the pressure, Eq (33), is then obtained from Eq. (32) after noting that the expansion coefficient at order $1 / N_{\tau}^{2 k}$ receives contributions from all terms in Eq. (32) with $n \leq k$ as $\Delta$ is a polynomial in $y / N_{\tau}$ starting at $\mathcal{O}\left(1 / N_{\tau}^{2}\right)$. Aside from a complicated factor that only depends on the angular variables $(\phi, \theta)$, these contributions are proportional to $y^{2 k}$. Irrespective of the structure of the dispersion relation of a particular action under consideration the contribution of the radial integral in Eq. (32) to an expansion coefficient at order $1 / N_{\tau}^{2 k}$ thus has the unique form

$$
\begin{aligned}
\int_{0}^{\infty} \mathrm{d} y y^{2 k+2+n}\left(\frac{\partial^{n-1} B(y, z)}{\partial y^{n-1}}+\frac{\partial^{n-1} B\left(y, z^{-1}\right)}{\partial y^{n-1}}\right) & =(-1)^{n-1} \frac{(2 k+2+n) !}{(2 k+3) !} \int_{0}^{\infty} \mathrm{d} y y^{2 k+3}\left(B(y, z)+B\left(y, z^{-1}\right)\right) \\
& =(-1)^{n} \frac{(2 k+2+n) !}{(2 k+4) !}(2 i \pi)^{2 k+4} B_{2 k+4}\left(\frac{\mu}{2 \pi T i}+\frac{1}{2}\right)
\end{aligned}
$$

The angular contribution is given by

$$
A_{2 k}=\frac{b_{4+2 k}}{4 \pi^{3}} \sum_{n=1}^{k} \frac{1}{n !} \int_{0}^{\pi} \mathrm{d} \theta \sin \theta \int_{0}^{2 \pi} \mathrm{d} \phi \sum_{\left(k_{1}, . . k_{n}\right)} \prod_{j=1}^{n} a_{k_{j}}(\phi, \theta),
$$

where the inner sum extends over all n-tuples of positive even integers with $\sum_{j=1}^{n} k_{j}=2 k$. Here we have introduced a multiplicative normalization factor, the Bernoulli number $b_{4+2 k}$, that arises from the integration of the radial variable $y$ at vanishing chemical potential and normalizes the polynomials $P_{2 k}(x)$ to unity for $x=0$.

For a few small values of the expansion parameter $k$ we give here the explicit form of the polynamials $P_{2 k}(x)$ defined 
in Eq. (34):

$$
\begin{aligned}
& P_{0}(x)=1+\frac{30}{7} x^{2}+\frac{15}{7} x^{4} \\
& P_{2}(x)=1+\frac{147}{31} x^{2}+\frac{105}{31} x^{4}+\frac{21}{31} x^{6} \\
& P_{4}(x)=1+\frac{620}{127} x^{2}+\frac{490}{127} x^{4}+\frac{140}{127} x^{6}+\frac{15}{127} x^{8} \\
& P_{6}(x)=1+\frac{12573}{2555} x^{2}+\frac{2046}{511} x^{4}+\frac{462}{365} x^{6}+\frac{99}{511} x^{8}+\frac{33}{255} x^{10}
\end{aligned}
$$

\section{APPENDIX B: THE PROPAGATOR FOR HYPERCUBE WILSON FERMIONS}

using the shorthand notation, $c_{i}=\cos a p_{i}$ and $s_{i}=\sin a p_{i}$ we write the the denominator of the fermion propagator for Wilson type fermions, Eq. (45), as

$$
D\left(p_{4}, \vec{p}\right)=\left(\frac{1}{4} \operatorname{Tr} \mathcal{K}_{1}^{2}+\kappa_{1}^{2}+\delta^{2}\right)+2 \cos k_{4}\left(\frac{1}{4} \operatorname{Tr} \mathcal{K}_{1} \mathcal{K}_{2}+\kappa_{1} \kappa_{2}\right)+\cos ^{2} k_{4}\left(\frac{1}{4} \operatorname{Tr} \mathcal{K}_{2}^{2}+\kappa_{2}^{2}-\delta^{2}\right)
$$

where

$$
\begin{aligned}
\kappa_{1}(\vec{p}) & =\lambda_{0}+2 \lambda_{1}\left(c_{1}+c_{2}+c_{3}\right)+4 \lambda_{2}\left(c_{1} c_{2}+c_{2} c_{3}+c_{3} c_{1}\right)+8 \lambda_{3} c_{1} c_{2} c_{3} \\
\kappa_{2}(\vec{p}) & =2 \lambda_{1}+4 \lambda_{2}\left(c_{1}+c_{2}+c_{3}\right)+8 \lambda_{3}\left(c_{1} c_{2}+c_{2} c_{3}+c_{3} c_{1}\right)+16 \lambda_{4} c_{1} c_{2} c_{3} \\
\delta(\vec{p}) & =2 \rho_{1}+4 \rho_{2}\left(c_{1}+c_{2}+c_{3}\right)+8 \rho_{3}\left(c_{1} c_{2}+c_{2} c_{3}+c_{3} c_{1}\right)+16 \rho_{4} c_{1} c_{2} c_{3}
\end{aligned}
$$

and the $\mathcal{K}_{i}$ matrices are given by

$$
\mathcal{K}_{1}=\sum_{i=1}^{3} \gamma_{i} \alpha_{i} \quad \mathcal{K}_{2}=\sum_{i=1}^{3} \gamma_{i} \beta_{i} .
$$

The coefficients appearing in this matrix representation are,

$$
\begin{aligned}
& \alpha_{1}(\vec{p})=2 s_{1}\left(\rho_{1}+2 \rho_{2}\left(c_{2}+c_{3}\right)+4 \rho_{3} c_{2} c_{3}\right) \\
& \alpha_{2}(\vec{p})=2 s_{2}\left(\rho_{1}+2 \rho_{2}\left(c_{3}+c_{1}\right)+4 \rho_{3} c_{3} c_{1}\right) \\
& \alpha_{3}(\vec{p})=2 s_{3}\left(\rho_{1}+2 \rho_{2}\left(c_{1}+c_{2}\right)+4 \rho_{3} c_{1} c_{2}\right) \\
& \beta_{1}(\vec{p})=4 s_{1}\left(\rho_{2}+2 \rho_{3}\left(c_{2}+c_{3}\right)+4 \rho_{4} c_{2} c_{3}\right) \\
& \beta_{2}(\vec{p})=4 s_{2}\left(\rho_{2}+2 \rho_{3}\left(c_{3}+c_{1}\right)+4 \rho_{4} c_{3} c_{1}\right) \\
& \beta_{3}(\vec{p})=4 s_{3}\left(\rho_{2}+2 \rho_{3}\left(c_{1}+c_{2}\right)+4 \rho_{4} c_{1} c_{2}\right)
\end{aligned}
$$

Finally, the expressions $P, Q$ and $R$ used in Eq.(45) and (49) are obtained as

$$
P(\vec{p})=\frac{1}{4} \operatorname{Tr} \mathcal{K}_{1} \mathcal{K}_{2}+\kappa_{1} \kappa_{2} \quad Q(\vec{p})=\frac{1}{4} \operatorname{Tr} \mathcal{K}_{2}^{2}+\kappa_{2}^{2}-\delta^{2} \quad R(\vec{p})=\frac{1}{4} \operatorname{Tr} \mathcal{K}_{1}^{2}+\kappa_{1}^{2}+\delta^{2}
$$

\section{APPENDIX C: THE EXPANSION COEFFICIENT $h_{6}$}

We here give the explicit result for the sixth order expansion coefficient $h_{6}$ appearing in Eq. (51), which has been obtained using Mathematica. It reads

$$
\begin{aligned}
h_{6}=\frac{-73}{8316} & -2288+734400 \lambda_{3}^{2}+470016 \lambda_{4}^{2}+\left(1175040-2585088 r+1410048 \rho^{2}\right) \lambda_{3} \lambda_{4} \\
& +82584 \rho_{2}-918432 \rho_{2}^{2}+288000 \rho_{3}-6846336 \rho_{2} \rho_{3}-12690432 \rho_{3}^{2}+247392 \rho_{4} \\
& -6345216 \rho_{2} \rho_{4}-23376384 \rho_{3} \rho_{4}-10685952 \rho_{4}^{2}+29376 \lambda_{2}^{2}(2-3 r)^{2}+1836 \lambda_{1}^{2}(1-2 r)^{2} \\
& -1762560 \lambda_{3}^{2} r-(940032-470016 r) \lambda_{4}^{2} r+1057536 \lambda_{3}^{2} r^{2}+5967 r^{3} \\
& +58752 \lambda_{2}(-2+3 r)\left(4 \lambda_{4}(-1+r)+\lambda_{3}(-5+6 r)\right) \\
& \left.+14688 \lambda_{1}(-1+2 r)\left(4 \lambda_{4}(-1+r)+\lambda_{2}(-2+3 r)+\lambda_{3}(-5+6 r)\right)\right)
\end{aligned}
$$




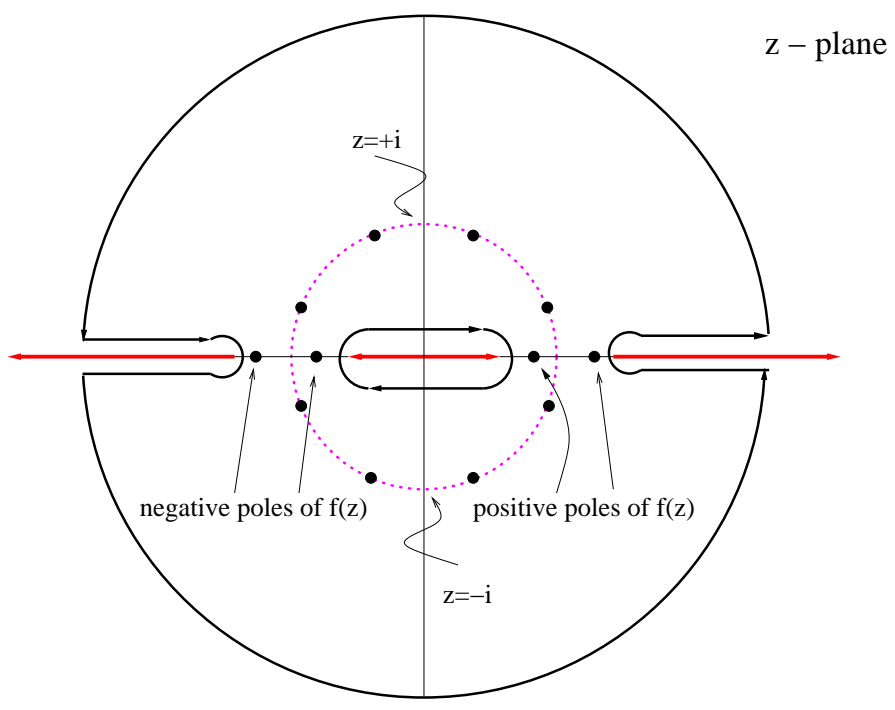

FIG. D.1: The locations of the poles and branch cuts of $H(z)$ and the choice of contour for $N_{\tau}=4$. The four poles of $f(z)$ only exist for $\alpha>0$. Also shown is the unit circle (dotted line) and the location of poles arising from the exponential integration kernel introduced in Eq. (D3).

where we have introduced the short hand notation,

$$
r=8 \rho_{2}+32 \rho_{3}+32 \rho_{4} .
$$

\section{APPENDIX D: MATSUBARA SUMS FOR OVERLAP FERMIONS}

We give here some further details on the evaluation of Matsubara sums appearing in calculations with overlap fermions. Throughout this appendix we make the dependence on the quark chemical potential explicit. We want to evaluate the sum

$$
\mathcal{S}(\alpha)=\frac{1}{N_{\tau}} \sum_{p_{4}} \ln \left(\sqrt{c^{2} x^{2}+b^{2}}-\alpha\right)
$$

where $x=\sin \left(\left(a p_{4}-i \mu a\right) / 2\right)$, and $b, c$ and $\alpha$ are functions of the three-momenta $\left(p_{1}, p_{2}, p_{3}\right)$ and $a p_{4}=2 \pi(n+1 / 2) / N_{\tau}$ with $n=0, \pm 1, \ldots, \pm\left(N_{\tau}-1\right) / 2, N_{\tau} / 2$.

To evaluate it, we first differentiate with respect to $\alpha$,

$$
\frac{\mathrm{d} \mathcal{S}}{\mathrm{d} \alpha}=-\frac{1}{N_{\tau}} \sum_{p_{4}} \frac{1}{\sqrt{c^{2} x^{2}+b^{2}}-\alpha} .
$$

This sum can be thought of as being part of the result of a contour integral over the function,

$$
H(z)=\frac{1}{\sqrt{-c^{2}\left(z-\frac{1}{z}\right)^{2} / 4+b^{2}}-\alpha} \frac{\mathrm{e}^{\mu a N_{\tau}}}{z\left(z^{2 N_{\tau}}+\mathrm{e}^{\mu a N_{\tau}}\right)}=f(z) \frac{\mathrm{e}^{\mu a N_{\tau}}}{z\left(z^{2 N_{\tau}}+\mathrm{e}^{\mu a N_{\tau}}\right)} .
$$

where the second equality defines the function $f(z)$. The function $H(z)$ has poles and cuts; the sum given in Eq. (D2) is related to the poles on the unit circle which arise from the last factor in the denominator of Eq. (D3). We want to evaluate the contour integral along the path shown in Fig. D. 1. As long as $\alpha \equiv \alpha(p)$ is positive there are four additional poles arising from $f(z)$ which are located at $\pm z_{0}$ and $\pm 1 / z_{0}$ with,

$$
z_{0}=e^{\tilde{x} / 2} \text { and } \cosh \tilde{x}=1+\frac{2}{c^{2}}\left(b^{2}-\alpha^{2}\right) .
$$

The residues at these points are listed in Table $\mathrm{D} 1$. 


\begin{tabular}{|r|c|}
\hline Pole: $z$ & Residue of $H(z)$ \\
\hline$z_{0}$ & $\frac{2 \alpha}{z_{0}^{2}-\left(1 / z_{0}\right)^{2}} \cdot \frac{1}{\left(z_{0}^{2} \mathrm{e}^{-\mu a}\right)^{N_{\tau}}+1}$ \\
$-z_{0}$ & $\frac{2 \alpha}{z_{0}^{2}-\left(1 / z_{0}\right)^{2}} \cdot \frac{1}{\left(z_{0}^{2} \mathrm{e}^{-\mu a}\right)^{N_{\tau}}+1}$ \\
$1 / z_{0}$ & $\frac{-2 \alpha}{z_{0}^{2}-\left(1 / z_{0}\right)^{2}} \cdot \frac{1}{\left(z_{0}^{-2} \mathrm{e}^{-\mu a}\right)^{N_{\tau}}+1}$ \\
$-1 / z_{0}$ & $\frac{-2 \alpha}{z_{0}^{2}-\left(1 / z_{0}\right)^{2}} \cdot \frac{1}{\left(z_{0}^{-2} \mathrm{e}^{-\mu a}\right)^{N_{\tau}}+1}$ \\
\hline
\end{tabular}

TABLE D.1: The four residues of $H(z)$ at the poles of $f(z)$ which exist for $\alpha>0$.

Moreover, there are branch cuts arising from the square root, which we take to be located in the intervals $C^{-}=$ $\left(-\infty, w_{0}\right), C^{0}=\left(-1 / w_{0}, 1 / w_{0}\right), C^{+}=\left(w_{0}, \infty\right)$, with $\sinh \left(w_{0}\right)=b / c$. We note that the contributions from cuts with $\operatorname{Re} z>0$ are identical to those with $\operatorname{Re} z<0$. Moreover, one can map the contribution from the positive part of the central cut, $\left(0,1 / w_{0}\right)$, onto the interval $C^{+}$. With this we arrive at the result,

$$
\begin{aligned}
\frac{\mathrm{d} \mathcal{S}}{\mathrm{d} \alpha}= & -\Theta(\alpha) \frac{4 \alpha}{c^{2} \sinh \tilde{x}}\left(\frac{1}{\mathrm{e}^{-N_{\tau}(\tilde{x}+\mu a)}+1}-\frac{1}{\mathrm{e}^{N_{\tau}(\tilde{x}-\mu a)}+1}\right) \\
& -\frac{2}{\pi} \operatorname{Im} \int_{w_{0}}^{\infty} \frac{f(z)}{z}\left(\frac{1}{\left(z^{2} \mathrm{e}^{-\mu a}\right)^{N_{\tau}}+1}-\frac{1}{\left(z^{-2} \mathrm{e}^{-\mu a}\right)^{N_{\tau}}+1}\right) .
\end{aligned}
$$

Integrating this again we obtain

$$
\begin{aligned}
\mathcal{S}(\alpha)=\quad & \Theta(\alpha) \frac{1}{N_{\tau}}\left(\ln \left(1+\mathrm{e}^{-N_{\tau}(\tilde{x}-\mu a)}\right)+\ln \left(1+\mathrm{e}^{-N_{\tau}(\tilde{x}+\mu a)}\right)+N_{\tau} \tilde{x}\right) \\
& +\frac{2}{\pi} \operatorname{Im} \int_{w_{0}}^{\infty} \mathrm{d} z \frac{\ln \left(\sqrt{-c^{2}\left(z-\frac{1}{z}\right)^{2} / 4+b^{2}}-\alpha\right)}{z}\left(\frac{1}{\left(z^{2} \mathrm{e}^{-\mu a}\right)^{N_{\tau}}+1}-\frac{1}{\left(z^{-2} \mathrm{e}^{-\mu a}\right)^{N_{\tau}}+1}\right)+\text { const. }
\end{aligned}
$$

It easily is seen that the absolute value of the integral appearing here is bounded by const $\cdot \exp \left(-N_{\tau} w_{0}\right)$. When we insert $S(\alpha)$ in Eq. (62) we thus find that only the first term in Eq. (D6) contributes to the large- $N_{\tau}$ expansion of the pressure.

[1] K. Symanzik, Nucl. Phys. B 226, 187 (1983) and Nucl. Phys. B 226, 205 (1983).

[2] S. Naik, Nucl. Phys. B 316, 238 (1989).

[3] F. Karsch, E. Laermann and A. Peikert, Nucl. Phys. B 605, 579 (2001).

[4] B. Sheikholeslami and R. Wohlert, Nucl. Phys. B 259, 572 (1985).

[5] P. Hasenfratz and F. Niedermayer, Nucl. Phys. B 414, 785 (1994);

P. Hasenfratz, S. Hauswirth, K. Holland, T. Jorg, F. Niedermayer and U. Wenger, Int. J. Mod. Phys. C 12, 691 (2001).

[6] W. Bietenholz and U.-J. Wiese, Nucl. Phys. B 464, 319 (1996).

[7] G. Boyd, J. Engels, F. Karsch, E. Laermann, C. Legeland, M. Lutgemeier and B. Petersson, Nucl. Phys. B 469, 419 (1996).

[8] B. Beinlich, F. Karsch, E. Laermann and A. Peikert, Eur. Phys. J. C 6, 133 (1999).

[9] C. R. Allton, S. Ejiri, S. J. Hands, O. Kaczmarek, F. Karsch, E. Laermann and C. Schmidt, Phys. Rev. D 68, 014507 (2003).

[10] C. Bernard et al. [MILC Collaboration], Phys. Rev. D 71, 034504 (2005) and Phys. Rev. D 77, 014503 (2008).

[11] W. Bietenholz and U.-J. Wiese, Phys. Lett. B 426, 114 (1998).

[12] C. Gattringer and L. Liptak, Phys. Rev. D 76, 054502 (2007).

[13] P. Hasenfratz and F. Karsch, Phys. Lett. B 125, 308 (1983).

[14] H.-Th. Elze, K. Kajantie and J. Kapusta, Nucl. Phys. B 304, 832 (1988).

[15] W. Bietenholz, R. Brower, S. Chandrasekharan and U.-J. Wiese, Nucl. Phys. Proc. Suppl. 53, 921 (1997).

[16] S. Shcheredin and E. Laermann, PoS LAT2006, 146 (2006).

[17] D.H. Adams, Nucl. Phys. (Proc. Suppl.) 129\&130, 513 (2004). 
[18] R. Narayanan and H. Neuberger, Phys. Lett. B 302, 62 (1993) and Nucl. Phys. B 443, 305 (1995).

[19] Y. Shamir, Nucl. Phys. B 406, 90 (1993).

[20] D. Kaplan, Phys. Lett. B 417, 141 (1998).

[21] for a recent review see: S. Capitani, Phys. Rept. 382, 113 (2003).

[22] G. Aarts and J. Foley [UKQCD Collaboration], JHEP 0702, 062 (2007).

[23] G. T. Fleming, Nucl. Phys. Proc. Suppl. 94, 393 (2001).

[24] H. Neuberger, Phys. Rev. D 57, 5417 (1998)

[25] R. G. Edwards and U. M. Heller, Phys. Rev. D 63, 094505 (2001).

[26] N. Christ, private communication 